\title{
Depleción del suministro de sangre y costo por donaciones indeterminadas del Hospital Nacional Guillermo Almenara Irigoyen
}

\author{
Jeel Moya-Salazar ${ }^{1, a}$, Liz Pio-Dávila ${ }^{2}$, Rommel Díaz $\mathrm{R}^{3}$
}

RESUMEN

Objetivo: Evaluar la depleción del suministro de sangre y el costo por donaciones indeterminadas del Hospital Nacional Guillermo Almenara Irigoyen durante el 2014.

Materiales y métodos: Estudio retrospectivo de corte transversal y análisis de costo-utilidad en donaciones con resultados en zona gris (valor de la muestra/ valor de corte entre los límites > $0.85 \mathrm{y}<1$ ) luego del tamizaje serológico para los siete marcadores infecciosos (HIV, HBsAg, HBcAb, HCV, HTLV-1/2, SíFILIS y CHAGAS) y la determinación en simultáneo del HIV Ag/Ab combo, por ELISA de cuarta generación. Desde el sistema e-Delphyn ${ }^{\circledR}$ se codificaron y tabularon los datos. El análisis de costos se realizó considerando el tipo de cambio vigente.

Resultados: De 9560 donaciones, el 20.7 \% (1977 donaciones) fueron resultados en zona gris lo que ocasionó una pérdida de 863.9 litros de sangre y 92640 USD. HbcAb con 10.18\% (973 resultados indeterminados) y anti-HIV con $0.39 \%$ (37 resultados indeterminados) fueron los resultados con mayor y menor seroprevalencia, respectivamente $(p<0.05)$. No se encontró diferencias significativas entre los marcadores anti-HIV y HIV Ag/Ab combo $(p=0.776)$.

Conclusiones: Se demostró que $>800$ litros de sangre descartada generó $>90$ mil USD de costo por donaciones indeterminadas ocasionando un perjuicio económico al presupuesto hospitalario y una disminución del suministro de sangre disponible para el tratamiento de pacientes en el Hospital Nacional Guillermo Almenara Irigoyen.

Palabras clave: Zona gris; donación de sangre; marcadores infecciosos; tamizaje.

\section{Depletion of blood supply and cost due to indeterminate donations at the Guillermo Almenara Irigoyen National Hospital}

\section{ABSTRACT}

Objective: To evaluate the depletion of blood supply and the cost due to indeterminate donations at Hospital Nacional Guillermo Almenara Irigoyen during 2014.

Materials and methods: A retrospective cross-sectional study and a cost-utility analysis were conducted in donations showing results in the gray zone (sample value/cutoff value between $>0.85$ and $<1$ ) after a serological screening of seven infectious markers (HIV, HBsAg, HBCAb, HCV, HTLV-1/2, syphilis and Chagas disease) and the simultaneous determination of the HIV Ag/Ab combo by means of a fourth generation ELISA. Data was encoded and tabulated using the e-Delphyn ${ }^{\circ}$ system. The cost-utility analysis was performed considering the current exchange rate.

Results: Out of 9,560 donations, $20.7 \%$ (1977 donations) showed results in the gray zone which caused a loss of 863.9 liters of blood and USD 92,640. The highest and lowest rate of seroprevalence were observed in $\mathrm{HBcAb}$ with $10.18 \%$ (973 indeterminate test results) and anti-HIV with $0.39 \%$ (47 indeterminate test results), respectively $(p<0.05)$. No significant differences were found between the anti-HIV y HIV Ag/Ab combo screening methods $(p=0.776)$.

Conclusions: It was demonstrated that more than 800 liters of discarded blood generated a more than USD 90,000 cost due to indeterminate donations causing economic damages to the hospital budget and a depletion of blood supply available for patients at Hospital Nacional Guillermo Almenara Irigoyen.

Keywords: Gray zone; blood donation, infectious markers; screening.

1. M.Sc(C). M.T., Departamento de Ciencias Celulares y Moleculares, Facultad de Ciencias y Filosofía. Universidad Peruana Cayetano Heredia Lima, Perú.

2. MLT. BSc., Hospital Nacional Dos de Mayo. Lima, Perú.

3. M.T. Especialista en control de calidad en bancos de sangre. EsSalud, Servicio de Banco de Sangre y Medicina Transfusional, Hospital Nacional Guillermo Almenara Irigoyen. Lima, Perú.

a. Hospital Nacional Docente Madre Niño San Bartolomé. Lima, Perú. 


\section{INTRODUCCIÓN}

La seguridad transfusional y de la donación son imperativos éticos de los bancos de sangre en el mundo entero. Estas instituciones deben de contener mecanismos de protección del tejido biológico que detecten noxas y disminuyan el riesgo transfusional logrando que esta práctica sea eficaz, segura e individualizada, y que la donación esté libre de reacciones adversas. Sin embargo, en la actualidad nuevas dificultades afectan a la seguridad del suministro de sangre: preguntas de selección de donantes poco estrictas, disponibilidad del suministro de sangre asequible, periodos de seroconversión, resultados indeterminados, agentes emergentes, entre otros ${ }^{(1,2)}$.

Las Infecciones Trasmisibles por vía Transfusional (ITT) son infecciones ocasionadas por virus, parásitos u otros potenciales patógenos que puede ser transmitido en la sangre donada, o en sus hemoderivados, a través de una transfusión ${ }^{(3)}$. Las ITT son difíciles de prevenir en los países en desarrollo, por ello el tamizaje de marcadores infecciosos constituye la medida definitiva para eliminar la sangre insegura ${ }^{(4)}$.

Actualmente, se disponen de pruebas de laboratorio con suficiencia para que los bancos de sangre tengan procedimientos fiables de tamiz serológico para los principales agentes infecciosos ${ }^{(5,6)}$. A pesar de los esfuerzos internacionales por desarrollar procesos de cribado serológico, el riesgo de transmisión de enfermedades infecciosas por vía sanguínea persiste, ciertamente pequeño, pero significativo.

El tamizaje en los bancos de sangre mediante serología convencional puede realizarse mediante Enzimoinmunoensayo (ELISA), hemoaglutinación indirecta ( $\mathrm{HAl}$ ), quimioluminiscencia (ECL por sus siglas en inglés), aglutinación de partículas (AP), entro otros métodos (7). $\mathrm{Si}$ bien el escrutinio sistemático de ITT utiliza ensayos confiables, diversos eventos ocasionan incertidumbre de medición y poca calidad de resultados. Usualmente el periodo de ventana implica un riesgo residual, los índices de infección y los riesgos relativos por transfusión heterogéneos y los resultados negativos cercanos al valor de corte (zona gris), pero que pudieran pertenecer a donantes seropositivos con una concentración muy baja que no puede ser detectado en estas pruebas ${ }^{(8)}$.

Además, tanto los resultados seropositivos como lo indeterminados de cada marcador ocasionan pérdidas de donaciones (incluyendo sus hemoderivados), aproximadamente de 457 litros de sangre por quinquenio, generando además una perdida global de costos institucional (61 984 USD por quinquenio), y disminuyendo la capacidad de donación del Servicio de Banco de Sangre de un Hospital Materno-Infantil de tercer nivel de atención en Lima, Perú ${ }^{(9,10,11)}$. La proporción de unidades eliminadas en los países de América Latina es $30.5 \%$ de unidades descartadas incluyendo todos los hemoderivados ${ }^{(12)}$.

Este estudio propone evaluar la depleción suministro de sangre y costo por donaciones indeterminadas del Hospital Nacional Guillermo Almenara Irigoyen durante el 2014. Además, se estimó la seroprevalencia de los marcadores infecciosos en unidades con resultado indeterminado.

\section{MATERIALES Y MÉTODOS}

\section{Tipo y diseño de investigación}

Se realizó un estudio retrospectivo de corte transversal y análisis de costo-utilidad básico. Este estudio fue justipreciado, corregido y aprobado por el comité externo de Ética e Investigación del Hospital Nacional Docente Madre Niño San Bartolomé.

\section{Población}

Toda donación (voluntaria, por reposición o pre-depósito) del servicio de Hemoterapia y Banco de Sangre del Hospital Nacional Guillermo Almenara Irigoyen durante el 2014 (Hospital Nivel III del Seguro Social - EsSalud).

\section{Muestra}

la muestra la confirmaron todas las donaciones con resultados indeterminados (resultados en zona gris) luego del tamizaje serológico para los siete marcadores infecciosos (anticuerpos contra el Virus de la Inmunodeficiencia Humana (HIV), antígeno superficial de la hepatitis B (HbsAg), antígeno core del virus de la hepatitis $B(H b c A b)$, anticuerpos contra el virus de la hepatitis C (HCV), anticuerpos contra los virus linfotrópicos humanos (HTLV$1 / 2$ ), anti-sífilis (SÍFILIS) y marcadores para la enfermedad de Chagas- Mazza (CHAGAS)) y la determinación en simultáneo del antígeno p24 y el anticuerpo contra HIV (HIV Ag/Ab combo) por enzimainmunoanálisis (ELISA) (Biokit, Lliça d'Amunt, Barcelona) de cuarta generación. Estos se seleccionaron respetando los criterios de donación establecidos por el Programa Nacional de Hemoterapia y Banco de Sangre (PRONAHEBAS) ${ }^{(13)}$.

Se consideró como zona gris la región en la que el resultado de la relación: valor de la muestra/ valor de corte (M/VC) se encontraba dentro de los límites $>0.85 \mathrm{y}<1$, es decir, que el resultado de la prueba era negativo, pero estaba muy cerca del valor de corte ${ }^{(8)}$. Para la definición de zona gris para cada marcador se consideraron las indicaciones del fabricante contenida en los reactivos.

Técnica de recolección de datos y procesamiento de la muestra

Los hemocomponentes de los donadores aparentemente sanos cuyos resultados estuvieron en zona gris de manera repetida se descartaron sin realizar pruebas confirmatorias. Estos resultados de tamizaje se registraron en libro de donaciones y el Sistema de Gestión de datos para bancos de sangre e-Delphyn ${ }^{\circledR}$ (Hemasoft, Singapur) del servicio, discriminados por edad, marcador serológico y absorbancia del ensayo inmunoenzimatico para cada marcador serológico. 
Se codificaron todos los resultados indeterminados para los siete marcadores infecciosos desde el sistema e-Delphyn ${ }^{\circledR}$ y/o el libro de donaciones del servicio, clasificándolos mensualmente hacia la Matriz de codificación en MS-Excel (Redmond, USA) durante el periodo de estudio.

Se evaluó la cantidad de sangre desecha por donante considerando los resultados indeterminados de $\geq 1$ marcador infeccioso para cada donación, la cual fue realizada íntegramente en bolsas cuádruples de donación TERUMO ${ }^{\circledR}$ (Shibuya, Tokio, Japón). El análisis de costoutilidad evaluó el costo de las unidades desechadas considerando el cambio de actual de Nuevos soles (NS) a Dólares americanos (USD) (3.36 NS = 1USD), siendo el costo unitario por unidad donada 50.6 USD (incluyendo escrutinio serológico, estudio completo del donador y bolsa cuádruple).

\section{Técnica de Análisis de Datos}

El análisis de datos se realizó en tres procesos básicos: codificación, tabulación y construcción de tablas y gráficos. La técnica utilizada para la verificación estadística de los resultados fue mediante el analizador estadístico IBM SPSS versión 20.0 (Armonk, USA) y MS-Excel. Se consideró valor de $p<0,05$ como estadísticamente significativo. Para la comparación de los resultados indeterminados para HIV con HIV Ag/Ab combo y anticuerpos contra HIV se utilizó la prueba de t de Students para muestras relacionadas.

Los aspectos éticos en la investigación están garantizados por la confiabilidad y el resguardo que tendrá el investigador sobre la información. Las principales limitaciones del estudio fueron: 1) que dado el objetivo del estudio no se consideraron los casos reactivos de los donantes durante el periodo de estudio, 2) no se tuvo acceso a la información detallada de los donantes durante el periodo de estudio 3) El banco de sangre no se encuentra acreditado bajo la norma ISO 15189. Pese a estas limitaciones se realizó por vez primera la evaluación de la depleción sanguínea y costo por unidades indeterminadas descartadas.

\section{RESULTADOS}

De 9560 donaciones realizadas durante el 2014, el 20,7 \% (1977 donaciones) fueron resultados indeterminados (en zona gris) para $\geq 1$ marcador serológicos tamizados durante el tiempo de estudio. La cantidad de muestras indeterminadas discriminadas por meses se muestran en la (Tabla 1).

Tabla 1. Proporción de muestras indeterminadas para marcadores infecciosos en Banco de Sangre durante el 2014

\begin{tabular}{|l|r|r|r|r|r|r|r|r|r|r|}
\hline & SIFILIS & HIV & HTLV-1/2 & HbsAg & HbcAb & HCV & CHAGAS & $\begin{array}{r}\text { HIV } \\
\text { Ag/Ab }\end{array}$ & Total \\
\hline Enero & 29 & 4 & 16 & 10 & 54 & 11 & 25 & 0 & 149 \\
\hline Febrero & 15 & 0 & 21 & 2 & 64 & 3 & 11 & 0 & 116 \\
\hline Marzo & 25 & 7 & 19 & 7 & 68 & 5 & 18 & 3 & 152 \\
\hline Abril & 19 & 11 & 33 & 5 & 71 & 3 & 21 & 8 & 171 \\
\hline Mayo & 35 & 7 & 17 & 8 & 86 & 9 & 16 & 12 & 190 \\
\hline Junio & 22 & 0 & 29 & 6 & 88 & 12 & 9 & 11 & 177 \\
\hline Julio & 28 & 0 & 21 & 8 & 96 & 8 & 20 & 4 & 185 \\
\hline Agosto & 31 & 1 & 9 & 5 & 64 & 2 & 7 & 0 & 119 \\
\hline Setiembre & 24 & 0 & 12 & 4 & 65 & 14 & 26 & 0 & 145 \\
\hline Octubre & 35 & 2 & 17 & 6 & 95 & 8 & 22 & 3 & 188 \\
\hline Noviembre & 29 & 5 & 10 & 9 & 151 & 12 & 19 & 4 & 239 \\
\hline Diciembre & 16 & 0 & 16 & 2 & 71 & 4 & 35 & 2 & 146 \\
\hline Total & 308 & 37 & 220 & 72 & 973 & 91 & 229 & 47 & 1977 \\
\hline
\end{tabular}

Nótese la cuantiosa proporción de muestras indeterminadas para los marcadores HbcAb y SIFILIS que ocasionaron una seroprevalencia de $10.18 \%$ y $3.22 \%$, respectivamente $(n=9560)$. 
La proporción de unidades indeterminadas, los costos y la cantidad de sangre perdida durante el periodo de estudio (Figura 1). El efecto directo de los resultados indeterminados luego del tamizaje serológico es la eliminación de unidades y todos sus hemoderivados, esto genera un reducción de la capacidad de donación del Banco de Sangre (por la cantidad de litros de sangre desechadas) e incrementa los costos institucionales por desecho de unidades tamizadas. Durante el 2014 se eliminaron 1832 unidades de sangre lo que representa una pérdida de sangre de $863.9 \pm 15$ Litros (rango: 50.7 a 104.4 litros). Del análisis de costo-utilidad se desprende que este desecho de unidades ocasiono un costo total anual de 311270 soles (92640 USD) solo por unidades indeterminadas ocasionando una depleción del suministro de sangre.

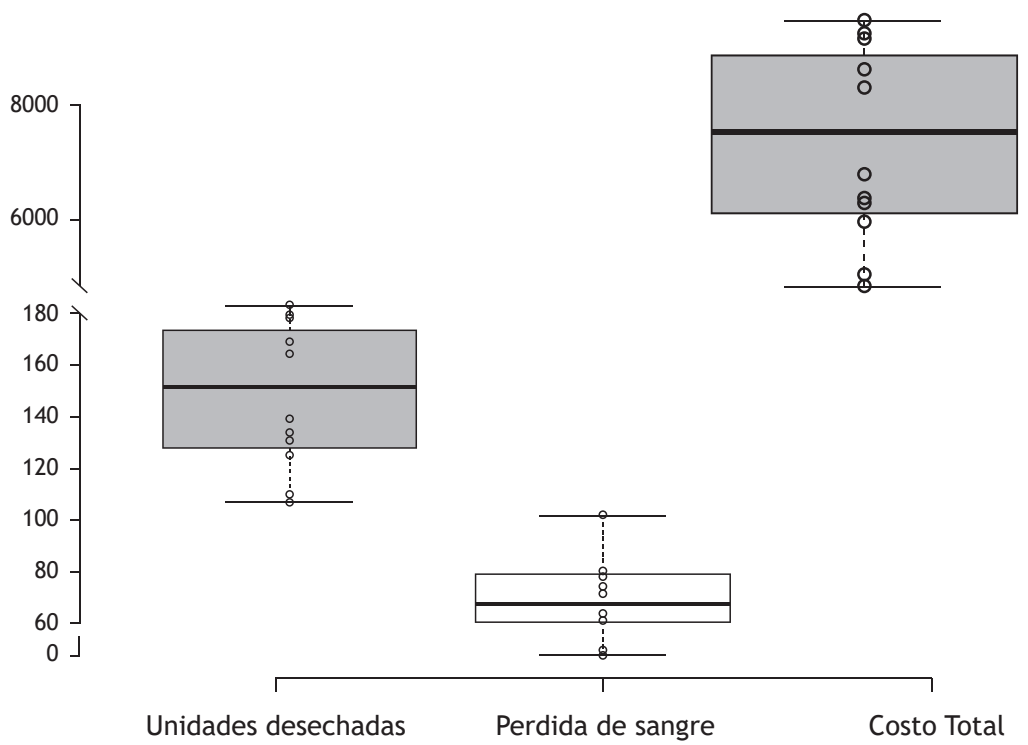

Figura 1. Proporción de unidades desechadas y costo por unidades indeterminadas durante el 2014 (distribución de puntos de datos por defecto)

El promedio de sangre desechada fue de $72 \pm 15$ litros (rango: 50.7 a 104.4 litros de sangre). El costo promedio fue de $7720 \pm 1386$ USD (rango: 5616.1 a 9461.3 USD). ( $n=9560)$.

La seroprevalencia de resultados indeterminados fue 20,7\%. En la (Figura 2) se muestra la seroprevalencia para cada marcador infeccioso (incluyendo HIV Ag/Ab combo). $\mathrm{HbcAb}$ con $10.18 \%$ (973 resultados indeterminados) y anti-HIV con $0.39 \%$ (37 resultados indeterminados) fueron los resultados con mayor y menor cuantía, respectivamente $(p<0,05)$. No se encontró diferencias significativas entre los marcadores anti-HIV y HIV Ag/Ab combo ( $p=0.776)$.

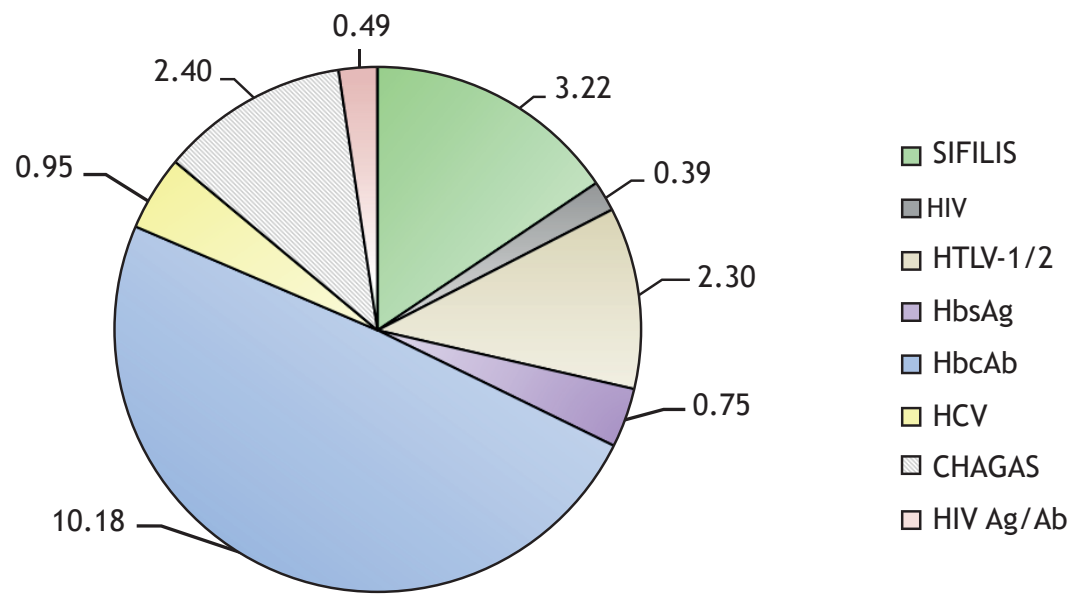

Figura 2. Seroprevalencia de marcadores infecciosos en resultados indeterminados del banco de sangre del Hospital Nacional Guillermo Almenara Irigoyen ( $=9560)$ 


\section{DISCUSIÓN}

Los resultados indeterminados (unidades con resultados en zona gris) para $\geq 1$ marcador infeccioso ocasionaron un costo > 90 mil USD y una pérdida de > 800 litros de sangre durante el 2014 en el Hospital Nacional Guillermo Almenara Irigoyen. Esto evidencia limitaciones en la cadena de donación que afectan el suministro de sangre de la institución.

En Latinoamérica, el 29\% de causas de descarte de unidades se debe a unidades reactivas para ITT, que no incluyen los resultados indeterminados o bordelinde, con lo cual el porcentaje podría incrementarse a un $50 \%{ }^{(12)}$. La cantidad de unidades desechadas y sangre perdida por donaciones indeterminadas en este estudio es muy consistente con los reportes sobre disponibilidad de componentes de la Organización Panamericana de la Salud $(11,3 \%)$ donde las mayores cantidades de hemocomponentes desechados son Plasma Congelado y Plasma Rico en Plaquetas, la cual es considerablemente mayor que lo reportado un quinquenio antes $(1,65 \%)^{(12,16)}$. La cantidad de sangre desechada por seropositividad en el Perú es de 457,2 litros (1016 donaciones por quinquenio), valores cercanos a lo desechado en otros bancos de sangre, como en La Habana ${ }^{(9,11)}$. Por el contrario, la cantidad de sangre desechada por resultados en zona gris (resultados indeterminados) es de 863,9 litros por año generando una cuantiosa depleción del suministro de sangre por el banco de sangre del Hospital (Figura 1). Se ha señalado que cuando se obtienen resultados de tamizaje para los marcadores infecciosos (como VIH y VHC) en zona gris, es necesario desechar la sangre y/o sus componentes donados como medida de seguridad, sobre todo ante el hecho de una posible infección temprana por estos virus $(2,8)$. La principal consecuencia del descarte de unidades con resultados en zona gris o indeterminada es el cuasi inconmensurable impacto económico que ocasiona. $\mathrm{Si}$ bien el costo por donación descartada para unidades seropositivas que analizamos con anterioridad en el Perú durante cinco años fue de 172720 soles (61893 USD), el originado por los resultados indeterminados genera un costo de 311270 soles (92640 USD) por año, superando reportes previos sobre donaciones reactivas en casi el doble ${ }^{(9,12,32)}$. Esto demuestra el gran impacto económico, sanitario y social de los bancos de sangre, asimismo representa un reto para todos los sistemas de aseguramiento de calidad y de la seguridad transfusional.

Además de lo señalado, la falta de donación voluntaria en nuestra comunidad es también un factor que ocasiona depleción en el suministro de unidades de sangre. Esta inmensa minoría de donación altruista ocasiona que se obtengan donaciones de poca calidad las cuales generan costos innecesarios e ineficientes durante el tamiz serológico y los procesos de manejo transfusional ${ }^{(18,19)}$.

Por otro lado, en nuestra región los indicadores de la disponibilidad y seguridad de la sangre y sus componentes para el Perú señalan un $100 \%$ de tamizaje para marcadores infecciosos que determinan una prevalencia global de 1,13\%. La prevalencia para marcadores infecciosos en Latinoamérica es de $0.73 \%$, siendo $\mathrm{HbcAb}$ y HIV los marcadores con mayor $(2,19 \%)$ y menor $(0,23 \%)$ seroprevalencia, respectivamente ${ }^{(12)}$. En el Perú estos porcentajes se mantienen constantes para los resultados reactivos ${ }^{(9,12)}$, así como también para los resultados indeterminados precisados en este estudio (Figura 2). Es de notar que la seroprevalencia global es de casi el doble de la regional, situándose el Perú el tercer país con mayor seroprevalencia global, por debajo de Paraguay $(1,88 \%)$ y Guatemala $(1,58 \%)^{(12)}$. Estos valores señalan la poca calidad de donantes, la alta prevalencia y el poco control sanitario-epidemiológico de las ITT ${ }^{(14)}$. Las estimaciones del riesgo de transmisión de enfermedades infecciosas por transfusión son esenciales en el monitoreo de la seguridad de la sangre donada y para la evaluación de los efectos potenciales de las nuevas pruebas de tamizaje ${ }^{(15)}$. Como ejemplo de comparación, Chile que desde el 2007 (0.13\%) al $2013(0.11 \%)$ a reducido su prevalencia ya que cuentan con una completa organización de sistema nacional que incluye sistemas de calidad y organismos de acreditación y certificación (Perú: 2007 (0,72\%) y $2013(1,13 \%)^{(12,16)}$.

Del total de resultados indeterminados $10.17 \%$ corresponden a marcadores para Hepatitis B, que con amplios factores de riesgo posee substancial significado en el cribado serológico de los bancos de sangre de la comunidad $^{(9,17)}$. La gran proporción de resultados en zona gris para marcadores anti-Sífilis señala la importancia del cribado de este marcador para los bancos de sangre, donde desarrolla un rol subrogante e importante en algunos hemocomponentes ${ }^{(18)}$. El riesgo de la transmisión de infecciones mediante la trasfusión de sangre, continúa existiendo, ciertamente pequeño pero significativo. La persistencia de este riesgo es debida a diferentes causas, fundamentalmente por la dificultad que los métodos de tamizaje serológico tienen para identificar a los donantes con infecciones recientes, la existencia de personas crónicamente infectadas y errores de laboratorio, tanto en las técnicas de tamizaje como en el procesamiento administrativo $^{(19)}$.

El riesgo de transmisión de Trypanosoma cruzi se incrementa en proporción a la prevalencia de donantes. En el Perú se tamiza este marcador por considerarse endémico al igual que otros países de la región. Esta infección representa un problema grave para la seguridad de la sangre. Determinamos $2.39 \%$ (229 resultados indeterminados) de resultados indeterminados para CHAGAS, resultados coincidentes con los últimos reportes de seroprevalencia ${ }^{(12)}$. La efectividad de la pruebas de tamizaje se ve afectadas por falta de pruebas confirmatorias, falta de estandarización de reactivos y reacciones cruzadas generando resultados indeterminados ${ }^{(9,20)}$. El HTLV-1/2 se conoce como agente causal de la paraparesia espástica tropical y leucemia de células T del adulto, entidad poco clara pero letal ${ }^{(21,22)}$. La distribución epidemiológica se caracteriza por agrupamiento geográfico, predominando la infección en lugares con alta prevalencia como en los países latinoamericanos donde es parte del tamizaje serológico. De todos estos países, el Perú presenta la más alta seroprevalencia para HTLV$1 / 2(0,88 \%)$, lo que también genera altas proporciones de 
resultados indeterminados en $11 \%$ del total de donaciones indeterminadas (220 donaciones) ${ }^{(12)}$. La determinación de resultados estaría sujeta, además, a la frecuencia y duración del periodo de ventana de este virus la cual es todavía desconocida ${ }^{(23)}$.

El HCV se contagia principalmente de manera ocupacional aunque las vías de contagio aun no estén establecidas, pero se plantea un mecanismo de transmisión mundial común ${ }^{(24)}$. La seroprevalencia mundial para HCV se estima en $1 \%$, sin considerar la ubicación geográfica, la práctica social o la genética, entonces la ejecución de pruebas de tamizaje serológico en banco de sangre para este marcador tiene un impacto significativo para la Salud Pública. En este estudio, determinamos que los resultados en zona gris para HVC (prevalencia de 0.95\%) superan los valores del promedio regional de seroprevalencia $(0,42 \%)$ y los reportes de resultados indeterminados con prueba confirmatoria $(0,12 \%)^{(8,9,12)}$. Según reportes anteriores solo $7 \%$ de resultados en zona gris tienen un resultado indeterminado cuando se aplica una prueba confirmatoria ${ }^{(8)}$; lo que hace necesario el seguimiento de estos donantes para conocer si se transforman en seropositivos ${ }^{(25)}$.

La epidemia del Síndrome de Inmunodeficiencia Humana (SIDA) ha aumentado exponencialmente en las últimas décadas exigiendo que los bancos de sangre utilicen métodos con mayor sensibilidad, como las tecnologías de ácidos nucleicos y los antígenos virales. Por varias razones, estos métodos no se han masificado exponencialmente en los laboratorios de tamizaje de la comunidad, motivo por el cual actualmente el aseguramiento de calidad de sangre indemne se realiza con pruebas convencionales como ELISAS o técnicas de Quimioluminiscencia ${ }^{(8,26,27)}$. El promedio regional de seroprevalencia para HIV es de $0,19 \%$, el promedio para el Perú es de $0,23 \%$ y recientemente comprobamos que una seroprevalencia de $0,19 \%$ se asoció directamente con sífilis ${ }^{(9,12)}$. En este estudio se determinó una seroprevalencia de $0.38 \%$ en unidades con resultados en zona gris para HIV (método anti-HIV), resultados discordantes con estudios anteriores en Latinoamérica donde sugieren resultados en zona gris muy pequeños para HIV $(0,04 \%)^{(8)}$. Al comparar dos metodologías de tamizaje de HIV (HIV Ag/Ab combo y anticuerpos contra HIV) no se encontraron diferencias significativas $(p=0.776)$. El HIV $\mathrm{Ag} / \mathrm{Ac}$ está diseñado para detectar antígeno p24 así como anticuerpos contra HIV-1, HIV-1 grupo 0 y HIV-2 ${ }^{(28)}$. Diversas evaluaciones entre estos métodos demuestran ventas y desventajas símiles, siendo importante su valoración concernientes a interferentes, rendimiento diagnóstico y costo-efectividad ${ }^{(29,30,31)}$. La variación en el rendimiento de cada método puede deberse al tamaño de análisis y al flujo de pacientes seropositivos durante este tiempo dado que se utilizaron por separado (Tabla 1).

El riesgo de transmisión de enfermedades infecciosas a través de las transfusiones es una grave dificultad para la seguridad de la sangre y la disponibilidad, sobre todo, en países pobres con bajo grado de desarrollo donde se requieren estrategias y compromisos de recursos innovadores, la aplicación de buenas prácticas en Medicina Transfusional, la promoción y el incremento de la donación voluntaria, la implementación de nuevas metodologías de tamizaje y el desarrollo de estrategias para reducir las donaciones inseguras.

En conclusión, este estudio evaluó la depleción de unidades de sangre y el alto costo ocasionado por unidades con resultado indeterminado (en zona gris) del Hospital Nacional Guillermo Almenara Irigoyen durante el 2014. Demostramos un desperdicio de $>800$ litros de sangre y un costo > 90 mil USD que generan un prejuicio económico al presupuesto hospitalario y una disminución del suministro de sangre disponible para el tratamiento de pacientes.

Este gran impacto socio-sanitario y económico podría evitarse mejorando la calidad de las pruebas de tamizaje para evitar resultados indeterminados (en zona gris), y promoviendo la donación voluntaria. Asimismo, dentro de los sistemas de aseguramiento de la calidad de cada institución se debe de evaluar estos determinantes con el fin de establecer procesos de mejora continua con el fin de asegurar la calidad de la práctica transfusional.

Agradecimientos: Expresamos nuestro agradecimiento a todos los integrantes del Servicio de banco de sangre y Medicina Transfusional del Hospital Nacional Guillermo Almenara Irigoyen en Lima, Perú; y un agradecimiento especial a la Dra. Jorgelina L. Blejer del Hemocentro de Buenos Aires, Argentina por la revisión juiciosa de este manuscrito.

\section{REFERENCIAS BIBLIOGRÁFICAS}

1. Goodnough LT, Shander A, Brencher ME. Transfusion medicine: looking to the future. Lancet 2003;361 (9352):161-9

2. Callery MF, Nevalainen DE, Kirst TM. Quality systems and total process control in blood banking. Transfusión.1994; 34 (10):899-906.

3. Choudhury N. Transfusion transmitted infections: How many more? Asian J Transfus Sci. 2010; 4(2): 71-72.

4. Vega MC, Montoro AJ. Manual de Medicina Transfusional. España: Mosby-Doyma; 1994.

5. Allan SR Jr. Administrative Manual Vol I, II, III. Bethesda, MD: American Association of Blood Banks. 1996.

6. Bihl F, Castelli D, Marincola F, Dodd R, Brander C. Transfusiontransmitted infections. J Transl Med. 2007; 5: 25.

7. Chiodini PL, Hartley S, Hewitt PE, Barbara JA, Lalloo K, Bligh $\mathrm{J}$, et al. Evaluation of a malaria antibody ELISA and its value in reducing potential wastage of red cell donations from blood donors exposed to malaria, with a note on a case of transfusion-transmitted malaria. Vox Sang. 1997;73(3):143148.

8. Aguirre GG, Martínez AJ, Arenas El. Resultados en zona gris del tamizaje para VIH y VHC, pueden indicar seroconversión inmediata. Rev Mex Patol Clin. 2005; 52(1): 63-68.

9. Moya SJ, Julcamanyan TE. Seroprevalence infectious markers causing loss of donations in the blood bank service in Hospital Nacional Docente Madre Niño San Bartolome 2008-2013. Horiz Med.2014; 14(4): 6-14.

10. Velásquez A, Cachay C, Munayco C, Poquioma E, Espinoza R, 
Seclén Y. La Carga de Enfermedad y Lesiones en el Perú. Perú: Ministerio de Salud; 2009.

11. Fano VR, Hernández PM, Jiménez DE, Longres MA. Marcadores serológicos causantes de pérdidas de donaciones. Rev Cubana Med Milit.2000;29(1):41-5.

12. Pan-American Health Organization. Supply of blood for transfusion in Latin American and Caribbean countries 2012 and 2013. Washington, DC : PAHO; 2015.

13. Ministerio de Salud. Sistema de Gestión de la Calidad del PRONAHEBAS. Manual de Calidad. Norma Técnica. No 011MINSA/ DGSP-V. 01. 2004.

14. Barbara JA. Challenges in transfusion microbiology. Transfus Med Rev. 1993;7(2):96-103.

15. Schreiber GB, Busch MP, Kleinman SH, Korelitz JJ. The risk of transfusion-transmitted viral infections. N Engl J Med.1996; 334:1685-1690

16. Pan American Health Organization. Supply of Blood for Transfusion in the Caribbean and Latin American Countries in 2006-2007: Progress since 2005 of the Regional Plan of Action for Transfusion Safety. Washington, DC : PAHO; 2009.

17. Sellors J, Zimic-Vincetic M, Howard M, Mahony JB, Chernesky MA. Predictors of positivity for hepatitis $B$ and the derivation of a selective screening rule in a Canadian sexually transmitted diseases clinic. J Clin Virol. 1998; 11(1):85-91.

18. Blejer JL, Carreras VL, Salamone HJ. Riesgo de Transmisión de Infecciones por Vía Transfusional. MEDICINA.2002; 62: 259-278

19. Grifols EJ, Martin VC, Hernández SJ, Pujol BM, Grifols RJ, García RE. Seguridad en Medicina Transfusional. Menarini Diagnóstics. España: Pecalo; 1998.

20. Blejer JL, Sartor PA, Botasso O, Salome HJ, Leguizamón MS. Trans-sialidase inhibition assay for the detection of Trypanosoma cruzi infection in blood donor samples from Argentina. Vox Sang.2008;95(3): 189-196.

21. Zur Hausen $H$. The search for infectious causes of human cancers: Where and why. Virology.2009;392 (1): 1-10

22. Khabbaz RF, Heneine W, Grindon A, Hartley TM, Shulman G, Kaplan J. Indeterminate HTLV serologic results in U.S. blood donors: are they due to HTLV-I or HTLV-II? J Acquir Immune Defic Syndr. 1992;5(4):400-4.

23. Dood RY. Hepatitis C virus, antibodies, and infectivity: parodox, pragmatism, and policy. Am J Clin Pathol. 1992; 97: 4-6.

24. Bush MP, Kleinman SH, Jackson B, Stramer SL, Hewlett I, Preston S. Nuclei acid amplification testing of blood donor for transfusion-transmitted infectious diseases: report of the interorganizational task force on nuclei acid amplification testing in blood donor. Transfusion 2000;40(2):143-159.
25. Melve GK, Myrmel H, Eide GE, Hervig T. Evaluation of the persistence and characteristics of indeterminate reactivity against hepatitis $\mathrm{C}$ virus in blood donors. Transfusion. 2009;49(11):2359-65.

26. Schoofs M. SIDA, la agonía de África. Merck Sharp \& Dohme. USA: The Village Voice; 2000.

27. Busch MP, Lee L, Satten GA, Henrard DR, Farzadegan H, Nelson $\mathrm{KE}$, et al. Time course of detection of viral and serologic markers preceding human immunodeficiency virus type 1 seroconversion: implications for screening of blood and tissue donors. Transfusion.1995; 35(2): 91-97.

28. Saville RD, Constantine NT, Cleghorn FR, Jack N, Bartholomew C, Edwards J, et al. Fourth Generation Enzyme-Linked Immunosorbent Assay for the Simultaneous detection of Human Immunodeficiency Virus Antigen and Antibody. J Clin Microbiol. 2001;39(7):2518-24.

29. Yilmaz G. Diagnosis of HIV infection and laboratory monitoring of its therapy. J Clin Virol. 2001 ; 21(3): 187-96.

30. Clinical and Laboratory Standards Institute (CLSI). Interference Testing in Clinical Chemistry; Approved Guideline - Second Edition. CLSI document EP7-A2. Wayne, PA: CLSI; 2005.

31. Beelaert G, Fransen K. Evaluation of a rapid and simple fourthgeneration HIV screening assay for qualitative detection of HIV p24 antigen and/or antibodies to HIV-1 and HIV-2. J Virol Methods. 2010; 168(1):218-22.

32. Sánchez AS, Gonzales NP, Alvarez VJ. Costos en la Transfusión Sanguínea. Rev Mex Anest. 2000;23(2): 66-70.

\section{Fuentes de financiamiento:}

Este artículo ha sido financiado por los autores.

\section{Conflictos de interés:}

Los autores declaran no tener ningún conflicto de interés.

\section{Correspondencia:}

Jeel Moya Salazar

Dirección: Jr. Pacifico 957. Lima 07. 51001 Lima, Perú.

Teléfono: +051 1986014954 .

Correo electrónico: jeelms@outlook.com; jeel.moya.s@pch.pe 